\title{
Resolution-enhanced OCT and expanded framework of information capacity and resolution in coherent imaging
}

Nichaluk Leartprapun

Cornell University

Steven Adie ( $\nabla$ sga42@cornell.edu )

Cornell University

\section{Research Article}

Keywords: optical coherence tomography, resolution, signal-to-noise ratio (SNR)

Posted Date: April 30th, 2021

DOI: https://doi.org/10.21203/rs.3.rs-444683/v1

License: (c) (1) This work is licensed under a Creative Commons Attribution 4.0 International License.

Read Full License 


\section{Abstract}

Spatial resolution in optical microscopy has traditionally been treated as a fixed parameter of the optical system. Here, we present an approach to enhance transverse resolution in beam-scanned optical coherence tomography (OCT) beyond its aberration-free resolution limit, without any modification to the optical system. Based on the theorem of invariance of information capacity, resolution-enhanced (RE)OCT navigates the exchange of information between resolution and signal-to-noise ratio (SNR) by exploiting efficient noise suppression via coherent averaging and a simple computational bandwidth expansion procedure. We demonstrate a resolution enhancement of $1.5 \times$ relative to the aberration-free limit while maintaining comparable SNR in silicone phantom. We show that RE-OCT can significantly enhance the visualization of fine microstructural features in collagen gel and ex vivo mouse brain. Beyond RE-OCT, our analysis in the spatial-frequency domain leads to an expanded framework of information capacity and resolution in coherent imaging that contributes new implications to the theory of coherent imaging. RE-OCT can be readily implemented on most OCT systems worldwide, immediately unlocking information that is beyond their current imaging capabilities, and so has the potential for widespread impact in the numerous areas in which OCT is utilized, including the basic sciences and translational medicine.

\section{Introduction}

The enhancement of resolution ${ }^{1-5}$ has been an important and on-going pursuit in all fields of imaging, including coherent ${ }^{6-27}$ and incoherent ${ }^{28-38}$ optical microscopy. One approach for resolution enhancement is spatial-frequency bandwidth expansion (i.e., increasing the numerical-aperture (NA)) via aperture synthesis. This family of techniques utilizes multiple measurements of the sample that provide access to different spatial frequencies beyond the bandwidth support of a single measurement. A wellknown technique in incoherent microscopy is structured illumination microscopy (SIM), which uses different illumination patterns to shift the spatial frequency coverage of the optical system ${ }^{32-34}$. In coherent microscopy, the bandwidth support of the optical system can be shifted via use of multiple illumination angles, as has been implemented with off-axis holography ${ }^{6-8}$ and Fourier ptychography ${ }^{9-11}$ (which performs incoherent imaging at different illumination angles, combined with phase retrieval methods to reconstruct the complex optical field). In optical coherence tomography (OCT), interferometric synthetic aperture microscopy (ISAM) utilizes synthetic aperture methods to overcome the trade-off between resolution and depth-of-field, in order to reconstruct depth-invariant focal-plane resolution from a single volumetric measurement with the optical focus at a fixed depth ${ }^{12,13}$. Combining full-field OCT with holography, holoscopy can similarly achieve focal-plane resolution across all depths ${ }^{14}$. More recently, optical coherence refraction tomography (OCRT) utilizes sample rotation combined with an (incoherent) Fourier synthesis technique reminiscent of X-ray computed tomography to effectively 'replace' the lateral resolution of a low-NA imaging beam by the superior axial resolution of $\mathrm{OCT}^{15}$. 
Another family of techniques aims to correct aberrations in order to restore ideal focal-plane resolution. Hardware-based adaptive optics (HAO) has been implemented in coherent ${ }^{16-19}$ and incoherent ${ }^{28-31}$ microscopy. In coherent imaging, access to the complex optical field can enable computational aberration correction post-data-acquisition. Computational adaptive optics (CAO) modifies the pupil phase of complex OCT tomograms to correct both defocus and optical aberrations, in order to restore aberration-free focal-plane resolution ${ }^{20-27}$. Indeed, reaching the ideal aberration-free resolution supported by the optical system (i.e., without entering the super-resolution regime), especially in complex media such as biological samples, is the aim of many adaptive optics or computational aberration correction methods in optical microscopy.

Spatial resolution (or equivalently, spatial-frequency bandwidth) in optical microscopy has traditionally been treated as a fixed parameter for a given optical system. However, the theorem of invariance of information capacity suggests that resolution of an optical system is a tuneable parameter that can be flexibly modified without altering the optical system ${ }^{3-5}$. Cox and Sheppard described the information capacity of an optical system as the product of its space-bandwidth products (SBP) over all spatial dimensions, time-bandwidth product (TBP), and its signal-to-noise ratio (SNR) on the logarithmic scale 4 . The theorem of invariance of information capacity states that it is not the spatial-frequency bandwidth (and therefore resolution), but the information capacity of an optical system that is invariant ${ }^{3-5}$. It follows that spatial resolution can, in theory, be enhanced beyond the aberration-free limit of a given optical system through an exchange of information between spatial-frequency bandwidth and SNR, while keeping the information capacity constant.

The framework of informational capacity and its invariance underscores several unique advantages of coherent over incoherent imaging. A coherent imaging system inherently supports twice the information capacity of an equivalent incoherent system because each pixel is described by both magnitude and phase of the complex optical field, as opposed to a single intensity value ${ }^{4,5,39}$. Using quantum Fisher information formalism, others have also shown that the resolution limit in traditional intensity-based imaging techniques can be overcome by making use of phase information ${ }^{40-42}$. Bilenca et al. derived the scattering limit to the information capacity of depth-resolved coherent imaging (using OCT as a case study) through turbid media as a function of $\mathrm{SNR}^{43}$. Furthermore, coherent averaging of complex tomograms provides a more efficient method for noise suppression than incoherent (magnitude-only) averaging in OCT, due to the decorrelation of random phase noise ${ }^{44-46}$. Coherent averaging has also been used for multiple scattering suppression ${ }^{47-49}$. The efficient noise suppression via coherent averaging presents an opportunity to expand the information capacity of a coherent imaging system via the enhancement of SNR.

Resolution-enhanced (RE)-OCT is a spatial-frequency bandwidth expansion approach that computationally enhances transverse spatial frequencies beyond the traditional bandwidth support (i.e., beyond the aberration-free resolution limit) of a beam-scanned OCT system. Unlike existing aperture synthesis techniques ${ }^{6-11,15,32-34}$, it does not rely on diversity in the illumination schemes to access 
traditionally unseen spatial frequencies, and so can readily be implemented on existing OCT systems. REOCT is based upon the premise of information exchange between spatial-frequency bandwidth and SNR, as governed by the theorem of invariance of information capacity ${ }^{3-5}$. It navigates this exchange of information by exploiting efficient noise suppression via coherent averaging and a simple computational bandwidth expansion procedure. RE-OCT harnesses the benefit of coherent averaging to (for the first time) enhance resolution in OCT.

In this paper we first discuss the underlying principle of RE-OCT based on the information capacity framework. Then, we demonstrate noise suppression via coherent averaging and analyse its impact on the OCT signal in not only the space, but also the spatial-frequency domain. Our analysis of the impact of coherent averaging in the transverse spatial-frequency domain provides a new perspective compared to prior work in OCT (which has only investigated the impact of coherent and incoherent averaging in the space domain $\left.{ }^{4-46}\right)$. Next, we demonstrate resolution enhancement by REOCT in both resolution phantom and biological samples (collagen gel and ex vivo mouse brain). We then analyse the factors that limit resolution enhancement in RE-OCT by comparing experimental results to simulations. Lastly, we leverage insights from our analysis in the spatial-frequency domain to present an expanded framework of information capacity and resolution in coherent imaging. RE-OCT has the potential to have widespread impact since it can be readily implemented on most OCT systems without requiring any redesign of the optical system or specialized computationally-intensive algorithms.

\section{Results}

\section{Underlying principle of resolution-enhanced OCT}

RE-OCT is a spatial-frequency bandwidth expansion approach based on the exchange of information between resolution and SNR that is supported by the underlying theorem of invariance of information capacity ${ }^{3-5}$. We conjecture that the transverse resolution of a tomogram acquired by a beam-scanned OCT system can be enhanced via computational bandwidth expansion (BE), where signal at higher spatial frequencies is raised via multiplication by a magnitude mask in the spatial-frequency domain (i.e., magnitude-based deconvolution). This is feasible due to the under-filling of the objective aperture (i.e., a physical bandwidth limit) that is implemented in the ubiquitous telecentric scanning scheme (Supplementary Fig. 2b) used by most OCT systems worldwide. However, deconvolution inherently amplifies noise and degrades the SNR of the image. To address this problem, we also conjecture that the SNR penalty associated with computational BE can be compensated by first suppressing the system noise-one efficient way in OCT is by coherently averaging multiple successively acquired complex tomograms ${ }^{44-46}$. The underlying principle of RE-OCT is illustrated in Fig. 1. RE-OCT utilizes coherent averaging for efficient noise suppression to 'earn' SNR, which can be used to 'purchase' resolution via computational BE.

To understand the underlying principle of RE-OCT in the context of the theorem of invariance of information capacity, we restate Cox and Sheppard's expression for the information capacity of an 
optical system ${ }^{4}$ (Eq. 1).

$$
C=\left(2 L_{x} B_{x}+1\right)\left(2 L_{y} B_{y}+1\right)\left(2 L_{z} B_{z}+1\right)\left(2 T B_{T}+1\right) \log _{2}(1+s / n),
$$

where $L, T$, and $B$ denote the spatial field-of-view (FOV), temporal duration, and bandwidth in the associated dimension, respectively. The first three terms represent the SBP along the three spatial dimensions while the fourth term represents the TBP. The last term represents the SNR (in bits), where $s$ and $n$ denote the average signal power and the additive noise power, respectively. Coherent averaging can be considered as the exchange of the temporal information (of an object that is invariant in time) for enhanced SNR. This effectively expands the information capacity of coherent-averaged versus the singleshot tomograms via the increase in the SNR term, which can be sacrificed to expand each of the transverse spatial-frequency bandwidths, $B_{x}$ and $B_{y}$. (For a full analysis of this process, see Supplementary Section I.) One scenario is to expand the bandwidth equally along each transverse dimension by a factor equal to the square root of the gain in SNR in order to, in principle, enhance resolution in the transverse plane by the same factor, without suffering any SNR penalty relative to the traditional single-shot tomogram (Supplementary Fig. 1). However, RE-OCT is not limited to this scenario; the trade-off between resolution and SNR can be flexibly navigated by tuning the number of tomograms averaged and the BE factor applied (i.e., more SNR than the amount 'earned' from coherent averaging can be sacrificed to prioritize further resolution enhancement) (Fig. 1a).

\section{Coherent-average noise suppression in space and spatial-frequency domains}

Noise suppression via coherent and incoherent average over $N=1$ through 100 acquisitions was investigated in both space and spatial-frequency domain in a silicone phantom containing scattering particles (Fig. 2). Supplementary Section III discusses the effects on the image signal in the space domain, where a coherent average demonstrated a factor of $\sqrt{ } N$ superior noise reduction efficiency over an incoherent average (Fig. 2a,b). Our results are consistent with previous work in OCT and theoretical trends ${ }^{45,46}$ (see Supplementary Section III for caveats of experimentally achieving the theoretical performance).

Here, we additionally investigated coherent-average noise suppression in the spatial-frequency domain (Fig. 2c-e), where the image is a superposition of the detected backscattered signal with a Gaussian magnitude spectrum (when imaged with a Gaussian beam) and the system noise with a uniform magnitude spectrum (circular Gaussian random variable in space). The power spectrum exhibited a dynamic range (DR) in the spatial-frequency domain, as measured from the power at $D C$ to the noise floor (Fig. 2d). A coherent average over $N=100$ acquisitions resulted in a suppressed noise floor that led to an increase in DR of $20 \mathrm{~dB}$, corresponding to noise reduction by a factor of 100 (Fig. 2d). In other words, the coherent average has revealed phase-stable but low-magnitude backscattered signal at higher spatial frequencies, which were originally below the noise floor in the single-shot image but are now above the suppressed noise floor. Consequently, signal phase remains correlated (i.e., absence of random phase 
variation) across spatial frequencies corresponding to a larger bandwidth (Fig. 2c,e). The improved correlation of signal phase after reduction in the noise floor can also be understood by considering the impact of SNR on phase noise in phase-sensitive $\mathrm{OCT}^{50,51}$. Supplementary Section VII investigates (in simulation) the relations between $N, D R$ and phase-correlation limit in the spatial-frequency domain.

\section{Resolution-enhanced OCT in silicone phantom}

Resolution enhancement in silicone phantom using coherent average over 100 acquisitions and a computational BE expansion factor of $2.4 \times$ is shown in Fig. 3. (See Methods and Supplementary Section IV for a complete description of the RE-OCT reconstruction procedure.) RE-OCT achieved a RE factor of $1.5 \times$, from the traditional aberration-free resolution of $2.1 \mu \mathrm{m}$ to an enhanced resolution of $1.4 \mu \mathrm{m}$ (Fig. $3 a, b$ ), while the peak signal-to-background ratio (SBR) marginally decreased from $50 \mathrm{~dB}$ to $48 \mathrm{~dB}$ (Fig. $3 a, c)$. However, when computational BE was performed on the single-shot image, not only was the SBR substantially decreased by $10 \mathrm{~dB}$, but the quality of the point spread function (PSF) also suffered (Fig. $3 a, c)$. This penalty is a result of computational BE indiscriminately amplifying both the backscattered signal and the system noise that dominates at higher spatial frequencies (Fig. 2d,e). Resolution improved at the cost of degraded SBR as a larger BE factor was applied (Fig. 3d,e and Supplementary Movie 1). Noise suppression prior to computational BE was essential in maintaining adequate SBR as well as the quality of the PSF in RE-OCT. However, even with a coherent average over 100 acquisitions, the best achievable resolution from this experiment was limited to $1.4 \mu \mathrm{m}$ at $\mathrm{BE}$ factor of 2.4 ; applying a larger $\mathrm{BE}$ factor only resulted in lower SBR and degraded PSF quality, without further improvement in resolution.

In order to investigate the factors that limit the experimentally achievable resolution enhancement, we performed the RE-OCT procedure on 6 simulated en face planes (3 conditions, each with and without aberrations) with properties representative of the silicone phantom images (Fig. 3d-g). (See Supplementary Section VI for information on the simulated en face planes.) Based on Cox and Sheppard's information capacity framework', the achieved RE factor is expected to be equivalent to the applied BE factor (see Supplementary Section VIII). This relationship holds true for the simulated ideal noise-free condition, in which the RE-OCT efficiency (defined as the ratio RE factor/BE factor) remained 1 up to the Nyquist limit (Fig. 3f, noise-free limit). However, the presence of system noise decreased the REOCT efficiency with increasing BE factor (Fig. 3f, noise only). The trends as a function of BE factor for resolution, SBR, and RE-OCT efficiency for the noise-only condition are remarkably consistent with the experimental results (Fig. $3 d-f$ ). Furthermore, the presence of scattering signal from the silicone background, in addition to system noise, caused a slight decrease in RE-OCT efficiency relative to the noise-only condition (Fig. 3f, noise with background). These results suggest that system noise is the primary limiting factor in RE-OCT. Indeed, both experiment and simulation showed that superior resolution was achieved with coherent average over larger $N$ (i.e., more noise suppression) for a BE factor of 2.4 (Fig. 3g). In addition, optical aberrations degraded resolution, SBR, and RE-OCT efficiency for all simulated conditions (Fig. 3d-g, red). As expected, the simulated condition incorporating all three contributions: system noise, silicone background, and aberrations, most closely matched the experiment. 


\section{Resolution-enhanced OCT in biological samples}

We implemented RE-OCT in collagen gel and ex vivo mouse brain, and show the best RE-OCT performances that were achieved, corresponding to BE factor of 2.0 (Figs. 4 and 5). In fibrous collagen gel, RE-OCT enhanced the visualization of the collagen fibre architecture by not only narrowing the width of the collagen fibres, but also increasing the peak signal magnitude of each fibre as a result of the resolution enhancement (Fig. 4a,b). Remarkably, low-contrast fine microstructural features, which were not clearly discernible in the traditional single-shot image due to weak signal, are more apparent in the RE-OCT image owing to the improved localization of signal energy in space (Fig. 4a, yellow arrows). In the BE single-shot image, the narrowing of fibre width can still be observed to a certain extent, but the peak signal magnitude did not improve as much (Fig. 4b). Furthermore, the SBR was degraded more severely in the BE single-shot image due to the amplification of noise without prior noise suppression (Fig. 4c). Computational BE with BE factors larger than 2.0 resulted in degraded SBR without further narrowing of the fibre width or improvement to the peak signal magnitude (Supplementary Movie 2), similar the degradation observed with BE factors larger than 2.4 in the silicone phantom (Supplementary Movie 1).

In ex vivo fresh mouse brain, RE-OCT enhanced the visualization of myelinated axonal processes, especially the low-contrast features that were less apparent in the traditional single-shot image (Fig. 5a, yellow arrows). Although the narrowing of fibre width could be observed in the BE single-shot image, the peak signal magnitude was degraded without coherent-average noise suppression (Fig. 5b), similar to the effects in collagen gel. Due to the typical fibre thickness of 1-3 $\mu \mathrm{m}$ (which is comparable to the native OCT transverse resolution of $2.1 \mu \mathrm{m}$ ) of myelinated axons ${ }^{52}$, some of the fibre narrowing observed here is not as prominent as in the collagen gel. The SNR penalty of the computational BE procedure is most apparent in the neuron (Fig. 5a, green inset), which produces lower OCT intensity than the surrounding brain tissue. Although the neuron remained visible in the RE-OCT image, the noise level in the BE singleshot image was brought up to that of the backscattered signal from the surrounding brain tissue, causing the neuron to 'disappear' into the background (Fig. $5 a, c)$. This emphasizes the importance of coherent averaging in RE-OCT, particularly when weak-scattering structures need to be clearly visualized. Computational BE with BE factors larger than 2.0 resulted in degraded SBR and lower contrast between the neurons and surrounding brain tissue, without further narrowing of the fibre width or improvement to the peak signal magnitude (Supplementary Movie 3).

\section{Factors that limit achievable resolution enhancement}

We revisit the image signal power and phase in the spatial-frequency domain (Fig. 2c-e) to further understand the role of system noise, background, and aberrations on RE-OCT resolution. System noise limits not only the available DR of the spatial-frequency-domain image, but also the spatial-frequency bandwidth over which signal phase (associated with any given point scatterer in space) remains correlated (Fig. 2d,e and Supplementary Fig. 7). Phase correlation in the spatial-frequency domain has a direct implication on the spatial resolution of a coherent image-in order to achieve the best localization 
of signal energy in space, signal at different spatial frequencies must be able to constructively interfere (i.e., be in-phase with each other). Thus, the phase-correlation limit (which is limited by SNR in our experiment) determines how much of the expanded spatial-frequency bandwidth (determined by the BE factor) can support constructive interference and contribute to enhancing the resolution in RE-OCT. Computational BE far beyond the phase-correlation limit only serves to amplify the contribution of phasedecorrelated higher spatial frequencies, which degrades the SBR and the quality of the PSF without further improving the resolution (Fig. $3 d-f$ and Supplementary Movie 1).

In contrast to system noise, background is composed of backscattered (single- (SS) and multiplescattering (MS)) signal from the sample medium (silicone in this case). The spatial-frequency spectrum of the SS background is bandlimited and obeys the imaging bandwidth support of the system (determined by the illumination beam width in our system). Meanwhile, evidence has shown that frequency content of MS background may extend beyond the imaging bandwidth of the system ${ }^{53}$. In either case, background may exhibit uncorrelated phase as opposed to a flat profile of an ideal PSF

(Supplementary Fig. 6b) and contribute to the disruption of phase correlation within (SS case) as well as outside (MS case) of the imaging bandwidth. As a result, resolution may be degraded by the presence of background compared to if the medium were completely transparent. In this respect, the role of background on OCT resolution is similar to that of optical aberrations-while aberrations contribute slowly varying phase inside the pupil, background contributes uncorrelated phase that results in the OCT speckle. Importantly, both effects imply that the sample itself may limit the achievable resolution; there can be contribution from sample-induced aberrations in addition to system aberrations, and the degradation of resolution by uncorrelated background phase becomes more severe when the structure of interest has lower SBR (e.g., due to weak scattering from the structure or strong scattering from the medium, or both). Furthermore, both background and aberrations are factors that cannot be mitigated by coherent-average noise suppression.

\section{Expanded framework of information capacity and resolution in coherent imaging}

A fundamental limit to resolution enhancement by RE-OCT is governed by the disruption of phase correlation in the spatial-frequency domain-due to system noise, background, aberrations, and other factors (e.g., sample instability, mechanical vibration, etc.). Among other factors, system noise played the most significant role in our experiments by determining the available DR of the image and the phasecorrelation limit in the spatial-frequency domain. Notably, system noise is also the only factor that can be suppressed via coherent averaging in our experiments. Thus, the basis of RE-OCT lies in navigating the trade-off between resolution (in the space domain) and DR (in the spatial-frequency domain) of the image via coherent-average noise suppression and computational BE (Fig. 1a), where DR represents the impact on SNR that manifests in the spatial-frequency domain (Fig. 2d). In Fig. 3a-c, we prioritized resolution enhancement and applied a BE factor of 2.4, which sacrificed more DR than the $20 \mathrm{~dB}$ earned with coherent averaging (Supplementary Fig.8b). Alternatively, we could apply a BE factor of only 1.4 and simultaneously improve both resolution and SBR by a smaller margin (Fig. 3d,e), where the SNR penalty 
was offset by the increased peak PSF intensity as a by-product of improved localization of the PSF in space (note the maxima in Fig. 3e).

In order to reconcile the predictions of information capacity and our experimental RE-OCT results, we propose an expanded framework of information capacity and resolution in coherent imaging (Fig. 6). The expanded framework emphasizes phase correlation in the spatial-frequency domain (in addition to SNR, FOV and spatial-frequency bandwidth in Cox and Sheppard's framework ${ }^{4}$ ) as an important facet of the information capacity of a coherent imaging system. In theory, resolution is governed by the imaging bandwidth of the optical system. In practice, however, phase-correlation limit in the spatial-frequency domain (Fig. 2e and Supplementary Fig. 7) must also be considered when determining the best achievable resolution. Supplementary Section VIII computes the resolution enhancement that is theoretically supported by Cox and Sheppard's information capacity framework ${ }^{4}$, and exemplifies the additional practical limit imposed by the SNR-limited phase-correlation limit (Supplementary Fig. 8a). Importantly, any factors-whether associated with the optical system or the sample itself-that can disrupt phase correlation in the spatial-frequency domain may prevent the optimal resolution (determined by the bandwidth support of the optical system) from being experimentally realized. By extension, in a time-dependent system whose information capacity includes the time-bandwidth product ${ }^{4}$, the temporal resolution of such a system would also be subjected to the correlation of phase in the temporal-frequency domain.

\section{Discussion}

Beam-scanning is by far the most common mode of acquisition in OCT. Most beam-scanned OCT systems utilize a Gaussian illumination beam that under-fills the physical aperture limit (e.g., objective lens) in the optical system. This under-filling is required for the widely used telecentric scanning scheme, which is implemented to minimize distortions due to coherence gate curvature. (Although ophthalmic OCT systems do not implement telecentric scanning, the typical beam diameter of $1.2 \mathrm{~mm}$ in standard clinical systems ${ }^{54}$ still under-fills the physical aperture of typical pupil diameters of adult eyes ${ }^{55}$ ). RE-OCT exploits this ubiquitous design feature in OCT systems to enable resolution enhancement beyond the aberration-free limit achieved by existing aberration correction approaches. In beam-scanned OCT, the aberration-free resolution limit is determined by a combination of the illumination beam width and the objective NA (Supplementary Section II); higher resolution is traditionally achieved by increasing the beam width or simply switching to a higher-NA objective (a standard practice in optical microscopy). RE$\mathrm{OCT}$, on the other hand, offers the flexibility to enhance transverse resolution beyond the aberration-free limit, using a simple computational procedure, without requiring any modification to the optical system or specialized acquisition schemes in existing aperture synthesis techniques. This gives RE-OCT a remarkable potential to have a widespread impact as it can be readily implemented on most OCT systems in the world and immediately unlock information that is beyond the current imaging capability of the system. 
Phase stability is vital for achieving optimal performance in RE-OCT, as is the case in other phasesensitive techniques in coherent imaging such as CAO and OCT angiography, which poses potential challenges for biological (e.g., live-cell imaging) and clinical (e.g., in vivo imaging) applications. In the case of RE-OCT, efficient earning of SNR via coherent-average noise suppression is contingent upon the backscattered signal from multiple acquisitions being phase-registered to each other. Our experiments in ex vivo fresh mouse brain (Fig. 5) required an additional image registration procedure (Supplementary Section V) to achieve phase registration before computing the coherent average. Others in OCT have also developed image registration and phase correction methods that successfully enabled phase-sensitive processing in biological samples, including for in vivo settings ${ }^{22,56-58}$. Furthermore, advances in highspeed imaging have enabled OCT imaging at $\mathrm{MHz}-$ rate $^{59}$, combatting motion artefacts and supporting phase-sensitive imaging in vivo ${ }^{24-26,46,60-62}$. RE-OCT may be particularly attractive for high-speed systems, some of which already incorporate coherent or incoherent averaging $46,60,61$, where the RE-OCT procedure can be easily integrated into the existing imaging workflow with minimal additional effort and imaging time. Thus, RE-OCT can draw from existing and emerging solutions in the field to address the challenges associated with achieving the required phase stability for biological and clinical applications, including in vivo imaging.

Beyond the potential applicability of RE-OCT for OCT systems worldwide, the concept that noise suppression (improved SNR) can be harnessed for resolution enhancement-where efficient noise suppression is key to 'purchasing' greater resolution enhancement-has broad implications for not only OCT but also optical microscopy and imaging science. Traditionally, averaging and noise suppression have been associated with the improvement of image contrast or SBR in optical microscopy. However, the theorem of invariance of information capacity suggests that there are opportunities to exploit the information gained via noise suppression in other facets of optical imaging. RE-OCT applies this concept to improve resolution through an exchange of information between spatial-frequency bandwidth and SNR.

Furthermore, the expanded framework of information capacity and resolution in coherent imaging presented here is broadly relevant and contributes to the theory governing coherent image formation. This includes coherent imaging with an aperture-filled system (e.g., full-field OCT) even though RE-OCT is only applicable when the physical aperture is under-filled. For instance, an image of a sample that generates particularly weak signal may have a very limited DR in the spatial-frequency domain, such that the SNRlimited phase-correlation limit is smaller than the imaging bandwidth of the optical system. Resolution would be limited by the available DR as opposed to the illumination NA (in an under-filled system) or the objective NA (in an aperture-filled system) in such a low-SNR scenario. Alternatively, the detected backscattered signal may be well above the noise floor, but both the object and the surrounding medium contribute comparable signal strength such that the DR spanned by the SS signal level in the sample is low. The disruption of phase correlation by the SS and MS background could limit the ability to resolve the object in such a low-DR scenario. By extension, physically increasing the image bandwidth of an optical system (e.g., by using a higher-NA objective or increasing the illumination beam width) would 
yield the optimal improvement in resolution only if the acquired image had sufficient DR in the spatialfrequency domain to support phase correlation over the increased bandwidth. Thus, our expanded framework highlights important practical considerations (associated with both the optical system and the sample/object) for resolution in all forms of coherent imaging.

Future development may combine RE-OCT with aberration-diverse $\mathrm{OCT}^{49}$ in order to suppress both the system noise and the MS background. Additionally, RE-OCT may be advantageous for imaging transversely isotropic structures (i.e., spatially invariant along a given spatial dimension) such as aligned muscle fibres or organized collagen fibrils in tendon and cartilage. This could allow for bandwidth along both the temporal and the invariant spatial dimension to be sacrificed to further enhance the resolution along the orthogonal spatial dimension ${ }^{3}$.

\section{Conclusion}

RE-OCT is an approach that offers the flexibility to enhance resolution in beam-scanned OCT beyond the aberrationfree resolution limit of the optical system. RE-OCT can be readily implemented on most OCT systems in the world without requiring any modification to the optical system. Based on the theorem of invariance of information capacity, RE-OCT navigates the information exchange between resolution and SNR by 'earning' SNR via coherentaverage noise suppression, in order to 'purchase' superior resolution via computational BE. Coherent averaging was shown to increase DR in the transverse spatial-frequency domain, and for the first time, has been harnessed for resolution enhancement in OCT. In silicone phantom, RE-OCT achieved a resolution improvement of $1.5 \times$ (NA of 0.2 to 0.3 ), while maintaining comparable SBR to the traditional single-shot image. In collagen gel and ex vivo mouse brain, RE-OCT significantly enhanced the visualization of fine microstructural features, including low-contrast features that were otherwise obscured in the traditional OCT image. We found that the phase-correlation limit represents an additional practical limit to the effective spatial-frequency bandwidth support of a coherent imaging system that can be more restrictive that the theoretical limit imposed by the existing theory of information capacity ${ }^{4}$. Based upon these insights, we presented an expanded framework of information capacity and resolution in coherent imaging to incorporate these factors. This framework emphasizes the fundamental role of phase correlation, which contributes important implications to the theory of coherent imaging.

\section{Methods}

\section{Optical system}

The optical system was a standard telecentric beam-scanned spectral-domain (SD)-OCT system (Supplementary Fig. 2a). The SD-OCT system was sourced by a broadband superluminescent diode with a central wavelength of $850 \mathrm{~nm}$ and a bandwidth of $120 \mathrm{~nm}$ (Superlum, M-T-850-HP-I). Spectral data was detected by a spectrometer with a bandwidth of $180 \mathrm{~nm}$ (Wasatch Photonics, Cobra 800) and a 2048pixel line-scan camera (e2v, Octopus). The sample arm utilized a double-pass illumination/collection 
configuration with an inverted 20x microscope objective with an NA of 0.45 (Olympus, LCPLN20XIR). Telecentric beam-scanning was accomplished with a 2-axis galvanometer and a zero-magnification telescope, which imaged the galvanometer to the back focal plane of the objective. The illumination beam diameter was $\sim 2-3 \mathrm{~mm}$, which under-filled the objective back aperture diameter of $8.1 \mathrm{~mm}$. The native transverse resolution was $2.1 \mu \mathrm{m}$ at the focal plane and the axial resolution was $1.9 \mu \mathrm{m}$ in air. The system sensitivity was $\sim 90 \mathrm{~dB}$ at the implemented acquisition rate (see RE-OCT image acquisition procedure) with a fall-off of $-5 \mathrm{~dB} / \mathrm{mm}$. The system was controlled by a custom-built LabVIEW acquisition software.

\section{Sample preparation}

All samples were prepared in glass coverslip-bottomed petri dishes, where the OCT beam interrogated the sample from the bottom through the coverslip (Supplementary Fig. 2c). The "noise image" for measuring the system noise in Fig. 2b,d was acquired by imaging the empty sample dish (Supplementary Fig. 2c).

Silicone phantom (Figs. 1 and 2) was prepared with a mixture of polydimethylsiloxane (PDMS) fluid (Clearco Product, PSF-50cst) and 2-part RTV silicone (Momentive Performance Materials, RTV-615 CLEAR $1 \#)$ at a weight ratio of 100:10:1 PDMS to RTV A to RTV B. Titanium dioxide particles with diameter of $0.5 \mu \mathrm{m}$ were dispersed as scattering particles. Silicone mixture was baked at $70{ }^{\circ} \mathrm{C}$ for at least 8 hours to complete the polymerization process. The sample was stored room temperature, where the temperature was allowed to stabilize, prior to imaging.

Collagen gel (Fig. 4) was prepared with type I collagen (Corning, Collagen I, rat tail) at a final collagen concentration of $2.0 \mathrm{mg} / \mathrm{mL}$. Collagen was polymerized at $4{ }^{\circ} \mathrm{C}$ for $15 \mathrm{~min}$., $20^{\circ} \mathrm{C}$ for $15 \mathrm{~min}$., and finally $37^{\circ} \mathrm{C}$ for $15 \mathrm{~min}$. to promote formation of heterogeneous fibre architecture with thick collagen fibres ${ }^{63}$. The sample was removed from incubation 1-2 hours before imaging to allow the temperature to stabilize at room temperature.

Ex vivo mouse brain (Fig. 5) was harvested post-mortem from a C57BL/6 mouse. Euthanasia was induced by an intraperitoneal injection of pentobarbitol $(150 \mu \mathrm{L}$ of $39 \mathrm{mg} / \mathrm{mL}$ solution in saline) and then perfused via intracardiac puncture with $30-\mathrm{mL}$ phosphate-buffered saline (PBS) at $4{ }^{\circ} \mathrm{C}$. The harvested brain was stored in PBS at $4{ }^{\circ} \mathrm{C}$ before embedded in $1 \%$ agarose (Sigma-Aldrich, Agarose, low gelling temperature) in the sample dish without fixation. The sample was kept at room temperature for 1-2 hours to allow the temperature to stabilize at room temperature before imaging. All animal procedures were approved by the Cornell Institutional Animal Care and Use Committee and were performed under the guidance of the Cornell Center for Animal Resources and Education. The study was carried out in compliance with the ARRIVE guidelines.

\section{RE-OCT image acquisition procedure}

Images were acquired in CM mode, where 3D OCT volumes were acquired successively to allow sufficient decorrelation of noise (see Supplementary Section III). Each volume was acquired with a line scan rate of 
$70 \mathrm{kHz}$, an exposure time of $10 \mu \mathrm{s}$, and a transverse spatial sampling of $0.4 \mu \mathrm{m} /$ pixel. In order to maximize the dynamic range spanned by the signal from the sample, image was acquired in the conjugated configuration by adjusting the reference arm such that the coverslip-bottom of the sample dish was positioned at larger pixel depths near the bottom of the B-scan (see Supplementary Section II).

\section{RE-OCT image reconstruction procedure}

RE-OCT image reconstruction from the acquired CM-mode volumes followed the procedure described in Supplementary Section IV. Briefly, space-domain OCT volumes were obtained from the raw tomograms via standard OCT image reconstruction, then, corrected for defocus via computational image formation procedures based on previously described methods ${ }^{64}$. For ex vivo mouse brain, an additional image registration procedure was required to correct bulk sample shift and phase drift in order to ensure that backscattered signal was spatially- and phase-registered across CM-mode volumes, as described in Supplementary Section V. Then, coherent average across processed OCT volumes was computed and its magnitude spectrum was obtained from the 2D transverse Fourier transform. A BE mask was computed from the magnitude spectrum at a given $B E$ factor and applied to the coherent-average OCT volume in the transverse spatial-frequency domain. Finally, the BE spectrum was zero-padded to upsample (in space) before returning to the space domain. The spatial upsampling was implemented to facilitate resolution measurement via curve-fitting to the PSF. All RE-OCT image reconstruction and subsequent image processing was performed in MATLAB R2017a. All OCT images shown has undergone defocus correction and represent the traditional aberration-free imaging capability before computational $\mathrm{BE}$.

\section{Calculations of OCT intensity}

The OCT intensity of the scattering particles, silicone background, and noise in Fig. 2b were computed as follows. Scattering particle intensity was obtained from the $99^{\text {th }}$ percentile of the OCT scattering intensity (i.e., square of OCT magnitude) of the space-domain image at the focal plane. Silicone background intensity was obtained from the median of the OCT scattering intensity of the particle-removed spacedomain image at the focal plane. The scattering particles were removed from the en face image via magnitude thresholding followed by a dilatation of the binary mask. Noise intensity was obtained from the standard deviation of the OCT scattering intensity of the "noise image" at the same pixel depth as the focal plane of the silicone phantom image. The "noise image" was obtained by imaging an empty blank sample dish (Supplementary Fig. 2c), placing the coverslip at the same pixel depth as in the silicone phantom.

\section{Calculation of dynamic range and phase-correlation limit}

The dynamic range in Fig. $2 \mathrm{~d}$ and Supplementary Fig. 7 was computed as follows. First, the noise power spectrum was obtained from the square of the magnitude of the 2D transverse Fourier transform of the "noise image" at the same pixel depth as the focal plane of the silicone phantom image. Then, the relative noise power was computed w.r.t. the signal power at DC (i.e., $k_{r}=0 \mathrm{rad} / \mu \mathrm{m}$ ) of the silicone 
phantom image at the focal plane. DR value in decibels was obtained from the mean of the relative noise power spectrum (uniformly distributed) across the entire transverse spatial-frequency domain.

The phase-correlation limit in Fig. 2e and Supplementary Fig. 7 was computed as follows. First, a window of size $243 \times 243$ pixels centred on a single scattering particle was cropped from the silicone phantom image at the focal plane. Then, the phase spectrum of the PSF was obtained from the angle of the 2D transverse Fourier transform of the window-out region. Next, local standard deviation of the phase spectrum was computed over a sliding kernel of size $3 \times 3$ pixels to obtain the "phase-decorrelation spectrum". The "phase-decorrelation spectrum" was divided into spatial-frequency bins, ranging from $k_{r}=$ $0 \mathrm{rad} / \mu \mathrm{m}$ to $k_{r}=7.85 \mathrm{rad} / \mu \mathrm{m}$ (the Nyquist limit) at bin width of $0.1 \mathrm{rad} / \mu \mathrm{m}$. Phase-correlation limit was obtained from the $k_{r}$ value at the centre of the bin at which the mean of the "phase-decorrelation spectrum" exceeded 0.2 rad.

\section{Measurements of resolution and SBR}

Resolution and SBR values in Fig. 3a,d-f were obtained from the Gaussian curve-fit to the PSFs (i.e., scattering particles) located at the focal plane. First, maximum intensity projection across 3 pixel-depths about the focal plane was computed from the OCT magnitude image. Scattering particles at the focal plane with peak magnitude $>5 \times 10^{4}$ were manually identified and the PSF images (a window of size 73 $\times 73$ pixels centred on each particle) were cropped out. Then, a total of 32 radial cross-sectional profiles of the PSF images (i.e., 1D PSF profiles at 32 different angular cross sections) were extracted for linear least-square curve fitting to a 1D Gaussian function. The fit parameters from the 32 cross-sectional profiles were averaged to obtain the peak magnitude and full width at half-maximum (FWHM) of each particle. At this stage, particles with FWHM $>2.4 \mu \mathrm{m}$ measured from the coherent-average OCT volume (i.e., noise-suppressed but not bandwidth-expanded) were excluded for being either air bubbles or aggregates of multiple particles. A total of 11 particles remained after the exclusion.

Resolution was obtained directly from the mean FWHM of the 11 remaining particles. SBR was obtained from the mean "peak SBR" of the 11 particles. The "peak SBR" in decibels of each particle was computed from the ratio of the peak PSF intensity (square of peak magnitude from the Gaussian fits described above) to the silicone background intensity (computed as described in Calculations of OCT intensity).

\section{Data availability}

Data underlying the results presented in this paper are not publicly available at this time but may be obtained from the authors upon reasonable request.

\section{Declarations}

Acknowledgements 
The authors would like to thank Dr Justin C. Luo and Dr David M. Small for preparing the collagen gel and harvesting the mouse brain specimen, respectively. This work is funded in part by National Institutes of Health (NIBIB-R01GM132823, Adie) and National Science Foundation (CAREER: CBET-1752405, Adie).

\section{Author Contributions}

N.L. prepared samples, performed all imaging experiments, developed RE-OCT reconstruction procedure, performed all data processing and analysis, and wrote the manuscript. S.G.A. conceived the principle of RE-OCT and provided guidance throughout this study. All authors reviewed and edited the manuscript.

\section{Additional Information}

See Supplementary Information for supporting content.

\section{Competing Interests}

The authors declare no competing interests.

\section{References}

1. den Dekker, A. J. \& van den Bos, A. Resolution: a survey. J. Opt. Soc. Am. A. 14, 547-557 (1997).

2. Cremer, C. \& Masters, B. R. Resolution enhancement techniques in microscopy. Eur. Phys. J. H. 38, 281-344 (2013).

3. Lukosz, W. Optical Systems with Resolving Powers Exceeding the Classical Limit. J. Opt. Soc. Am. 56, 1463-1472 (1966).

4. Cox, I. J. \& Sheppard, C. J. R. Information capacity and resolution in an optical system. J. Opt. Soc. Am. A. 3, 1152-1158 (1986).

5. Sheppard, C. J. R. \& Larkin, K. G. Information capacity and resolution in three-dimensional imaging. Optik. 113, 548-550 (2003).

6. Alexandrov, S. A., Hillman, T. R. \& Gutzler, T. \& Sampson, D. D. Synthetic aperture fourier holographic optical microscopy. Phys. Rev. Lett. 97, 168102 (2006).

7. Mico, V., Zalevsky, Z., García-Martínez, P. \& García, J. Synthetic aperture superresolution with multiple off-axis holograms. J. Opt. Soc. Am. A. 23, 3162-3170 (2006).

8. Luo, W., Greenbaum, A., Zhang, Y. \& Ozcan, A. Synthetic aperture-based on-chip microscopy. Light Sci. Appl. 4, e261 (2015).

9. Zheng, G., Horstmeyer, R. \& Yang, C. Wide-field, high-resolution Fourier ptychographic microscopy. Nat. Photonics. 7, 739-745 (2013).

10. Tian, L., Li, X., Ramchandran, K. \& Waller, L. Multiplexed coded illumination for Fourier Ptychography with an LED array microscope. Biomed. Opt. Express. 5, 2376-2389 (2014). 
11. Tian, L. et al. Computational illumination for high-speed in vitro Fourier ptychographic microscopy. Optica. 2, 904-911 (2015).

12. Ralston, T. S., Marks, D. L., Carney, P. S. \& Boppart, S. A. Interferometric synthetic aperture microscopy. Nat. Phys. 3, 129-134 (2007).

13. Davis, J. B. et al. Nonparaxial vector-field modeling of optical coherence tomography and interferometric synthetic aperture microscopy. J. Opt. Soc. Am. A. 24, 2527-2542 (2007).

14. Hillmann, D., Franke, G., Luhrs, C., Koch, P. \& Huttmann, G. Efficient holoscopy image reconstruction. Opt. Express. 20, 21247-21263 (2012).

15. Zhou, K. C., Qian, R., Degan, S., Farsiu, S. \& Izatt, J. A. Optical coherence refraction tomography. Nat. Photonics. 13, 794-802 (2019).

16. Hermann, B. et al. Adaptive-optics ultrahigh-resolution optical coherence tomography. Opt. Lett. 29, 2142-2144 (2004).

17. Zhang, Y., Rha, J., Jonnal, R. S. \& Miller, D. T. Adaptive optics parallel spectral domain optical coherence tomography for imaging the living retina. Opt. Express. 13, 4792-4811 (2005).

18. Zawadzki, R. J. et al. Adaptive-optics optical coherence tomography for high-resolution and highspeed 3D retinal in vivo imaging. Opt. Express. 13, 8532-8546 (2005).

19. Zhang, Y. et al. High-speed volumetric imaging of cone photoreceptors with adaptive optics spectraldomain optical coherence tomography. Opt. Express. 14, 4380-4394 (2006).

20. Adie, S. G., Graf, B. W., Ahmad, A., Carney, P. S. \& Boppart, S. A. Computational adaptive optics for broadband optical interferometric tomography of biological tissue. Proc. Natl. Acad. Sci. U.S.A. 109, 7175-7180(2012).

21. Ahmad, A. et al. Real-time in vivo computed optical interferometric tomography. Nat. Photonics. 7, 444-448 (2013).

22. Shemonski, N. D. et al. Computational high-resolution optical imaging of the living human retina. Nat. Photonics. 9, 440-443 (2015).

23. Kumar, A. et al. Anisotropic aberration correction using region of interest based digital adaptive optics in Fourier domain OCT. Biomed. Opt. Express. 6, 1124-1134 (2015).

24. Ginner, L. et al. Noniterative digital aberration correction for cellular resolution retinal optical coherence tomography in vivo. Optica. 4, 924-931 (2017).

25. Hillmann, D. et al. Aberration-free volumetric high-speed imaging of in vivo retina. Sci. Rep. 6, 35209 (2016).

26. Ginner, L. et al. Holographic line field en-face OCT with digital adaptive optics in the retina in vivo. Biomed. Opt. Express. 9, 472-485 (2018).

27. Hillmann, D. et al. Computational adaptive optics for optical coherence tomography using multiple randomized subaperture correlations. Opt. Lett. 44, 3905-3908 (2019).

28. Booth, M. J., Neil, M. A., Juskaitis, R. \& Wilson, T. Adaptive aberration correction in a confocal microscope. Proc. Natl. Acad. Sci. U.S.A. 99, 5788-5792(2002). 
29. Roorda, A. et al. Adaptive optics scanning laser ophthalmoscopy. Opt. Express. 10, 405-412 (2002).

30. Rueckel, M., Mack-Bucher, J. A. \& Denk, W. Adaptive wavefront correction in two-photon microscopy using coherence-gated wavefront sensing. Proc. Natl. Acad. Sci. U.S.A. 103, 17137-17142(2006).

31. Zheng, W. et al. Adaptive optics improves multiphoton super-resolution imaging. Nat. Methods. 14, 869-872 (2017).

32. Gustafsson, M. G. L. Surpassing the lateral resolution limit by a factor of two using structured illumination microscopy. J. Microsc. 198, 82-87 (2000).

33. Schermelleh, L. et al. Subdiffraction Multicolor Imaging of the Nuclear Periphery with 3D Structured Illumination Microscopy. Science. 320, 1332-1336 (2008).

34. York, A. G. et al. Resolution doubling in live, multicellular organisms via multifocal structured illumination microscopy. Nat. Methods. 9, 749-754 (2012).

35. Hell, S. W. \& Wichmann, J. Breaking the diffraction resolution limit by stimulated emission: stimulated-emission-depletion fluorescence microscopy. Opt. Lett. 19, 780-782 (1994).

36. Schneider, J. et al. Ultrafast, temporally stochastic STED nanoscopy of millisecond dynamics. Nat. Methods. 12, 827-830 (2015).

37. Rust, M. J., Bates, M. \& Zhuang, X. Sub-diffraction-limit imaging by stochastic optical reconstruction microscopy (STORM). Nat. Methods. 3, 793-795 (2006).

38. Huang, B., Wang, W., Bates, M. \& Zhuang, X. Three-Dimensional Super-Resolution Imaging by Stochastic Optical Reconstruction Microscopy. Science. 319, 810-813 (2008).

39. Fellgett, P. B. \& Linfoot, E. H. On the assessment of optical images. Proc. R. Soc. London Ser. A 247, 369-407(1955).

40. Tsang, M., Nair, R. \& Lu, X. M. Quantum Theory of Superresolution for Two Incoherent Optical Point Sources. Phys. Rev. X. 6, 031033 (2016).

41. Paúr, M., Stoklasa, B., Hradil, Z., Sánchez-Soto, L. L. \& Rehacek, J. Achieving the ultimate optical resolution. Optica. 3, 1144-1147 (2016).

42. Tham, W. K., Ferretti, H. \& Steinberg, A. M. Beating Rayleigh's Curse by Imaging Using Phase Information. Phys. Rev. Lett. 118, 070801 (2017).

43. Bilenca, A., Lasser, T., Bouma, B. E., Leitgeb, R. A. \& Tearney, G. J. Information Limits of Optical Coherence Imaging Through Scattering Media. IEEE Photonics J. 1, 119-127 (2009).

44. Szkulmowski, M. \& Wojtkowski, M. Averaging techniques for OCT imaging. Opt. Express. 21, 97579773 (2013).

45. Baumann, B. et al. Signal averaging improves signal-to-noise in OCT images: But which approach works best, and when? Biomed. Opt. Express. 10, 5755-5775 (2019).

46. Pfeiffer, T. et al. Flexible A-scan rate MHz-OCT: efficient computational downscaling by coherent averaging. Biomed. Opt. Express. 11, 6799-6811 (2020).

47. Kang, S. et al. Imaging deep within a scattering medium using collective accumulation of singlescattered waves. Nat. Photon. 9, 253-258 (2015). 
48. Thrane, L. et al. Complex decorrelation averaging in optical coherence tomography: a way to reduce the effect of multiple scattering and improve image contrast in a dynamic scattering medium. Opt. Lett. 42, 2738-2741 (2017).

49. Liu, S., Lamont, M. R. E., Mulligan, J. A. \& Adie, S. G. Aberration-diverse optical coherence tomography for suppression of multiple scattering and speckle. Biomed. Opt. Express. 9, 4919-4935 (2018).

50. Park, B. H. et al. Real-time fiber-based multi-functional spectral-domain optical coherence tomography at 1.3 um. Opt. Express. 13, 3931-3944 (2005).

51. Chang, E. W., Kobler, J. B. \& Yun, S. H. Subnanometer optical coherence tomographic vibrography. Opt. Lett. 37, 3678-3680 (2012).

52. Kwon, J. et al. Label-free nanoscale optical metrology on myelinated axons in vivo. Nat. Commun. 8 , 1832 (2017).

53. Chowdhury, S. et al. High-resolution 3D refractive index microscopy of multiple-scattering samples from intensity images. Optica. 6, 1211 (2019).

54. Reddikumar, M., Tanabe, A., Hashimoto, N. \& Cense, B. Optical coherence tomography with a 2.8-mm beam diameter and sensorless defocus and astigmatism correction. J. Biomed. Opt. 22, 26005 (2017).

55. Spector, R. H. in The History, Physical, and Laboratory Examinations (eds H.K. Walker, W. D. Hall, \& J. W. Hurst) Ch. 58(Butterworths, 1990).

56. Vakoc, B. J. et al. Three-dimensional microscopy of the tumor microenvironment in vivo using optical frequency domain imaging. Nat. Med. 15, 1219-1223 (2009).

57. Lee, J., Srinivasan, V., Radhakrishnan, H. \& Boas, D. A. Motion correction for phase-resolved dynamic optical coherence tomography imaging of rodent cerebral cortex. Opt. Express. 19, 21258-21270 (2011).

58. Oikawa, K., Oida, D., Makita, S. \& Yasuno, Y. Bulk-phase-error correction for phase-sensitive signal processing of optical coherence tomography. Biomed. Opt. Express. 11, 5886-5902 (2020).

59. Klein, T. \& Huber, R. High-speed OCT light sources and systems [Invited]. Biomed. Opt. Express. 8, 828-859 (2017).

60. Fechtig, D. J. et al. Line-field parallel swept source MHz OCT for structural and functional retinal imaging. Biomed. Opt. Express. 6, 716-735 (2015).

61. Kim, T. S. et al. 9.4 MHz A-line rate optical coherence tomography at $1300 \mathrm{~nm}$ using a wavelengthswept laser based on stretched-pulse active mode-locking. Sci. Rep. 10, 9328 (2020).

62. Kolb, J. P. et al. Live video rate volumetric OCT imaging of the retina with multi-MHz A-scan rates. PLoS One. 14, e0213144 (2019).

63. Mulligan, J. A., Ling, L., Leartprapun, N., Fischbach, C. \& Adie, S. G. Computational 4D-OCM for labelfree imaging of collective cell invasion and force-mediated deformations in collagen. Sci. Rep. 11, 2814 (2021). 
64. Mulligan, J. A., Feng, X. \& Adie, S. G. Quantitative reconstruction of time-varying 3D cell forces with traction force optical coherence microscopy. Sci. Rep. 9, 4086 (2019).

\section{Figures}

a Computational BE: increasing BE factor
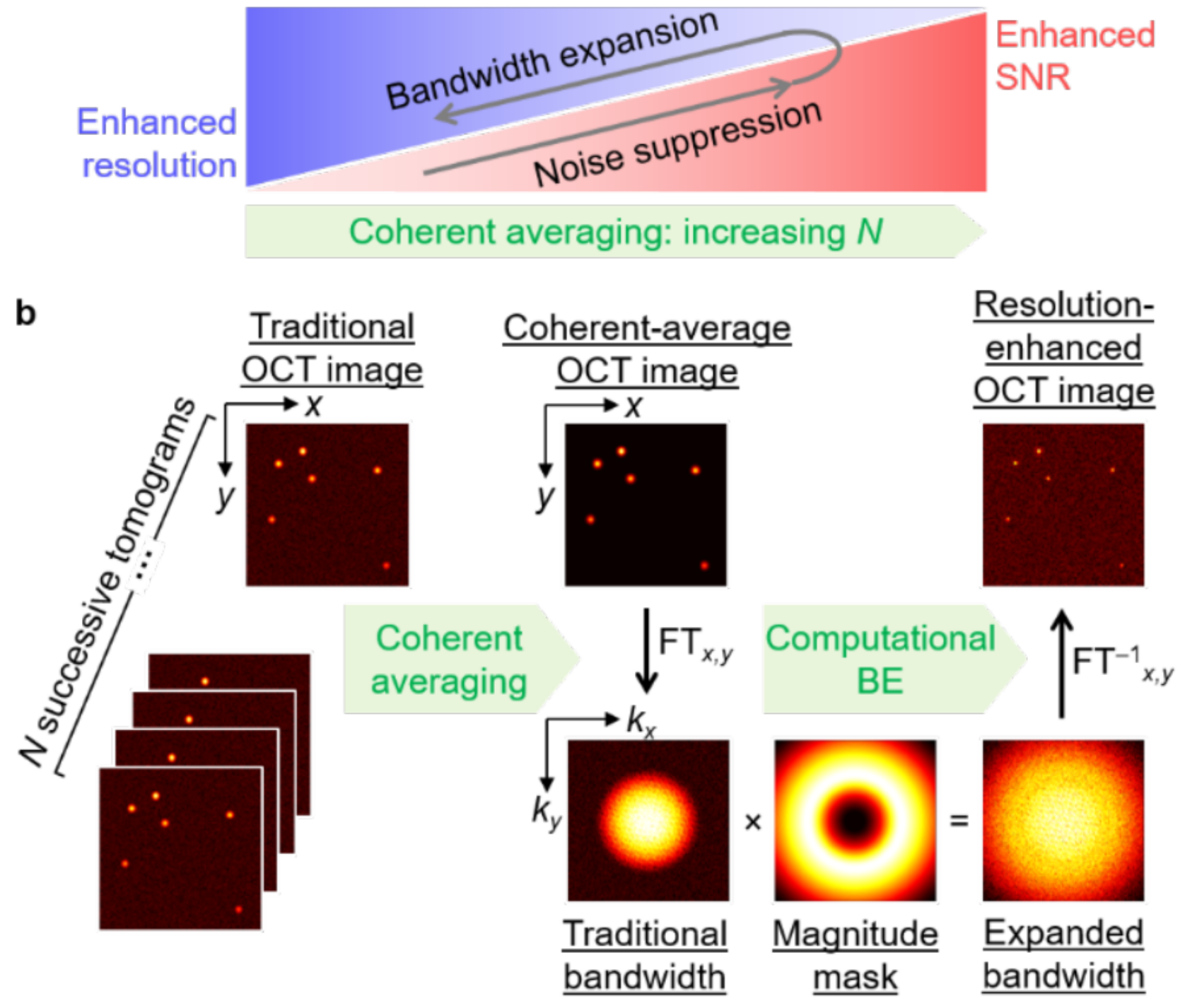

Figure 1

Underlying principle of RE-OCT. a, Information exchange between resolution and SNR facilitated by noise suppression and spatial-frequency bandwidth expansion. RE-OCT utilizes coherent averaging to efficiently suppress noise and enhance SNR, then SNR is sacrificed in the computational BE procedure to enhance resolution. b, Illustration of RE-OCT process to implement the principle in a. Supplementary Section IV provides a full description of RE-OCT reconstruction procedure. BE, bandwidth expansion. $\mathrm{FT}$, Fourier transform. 

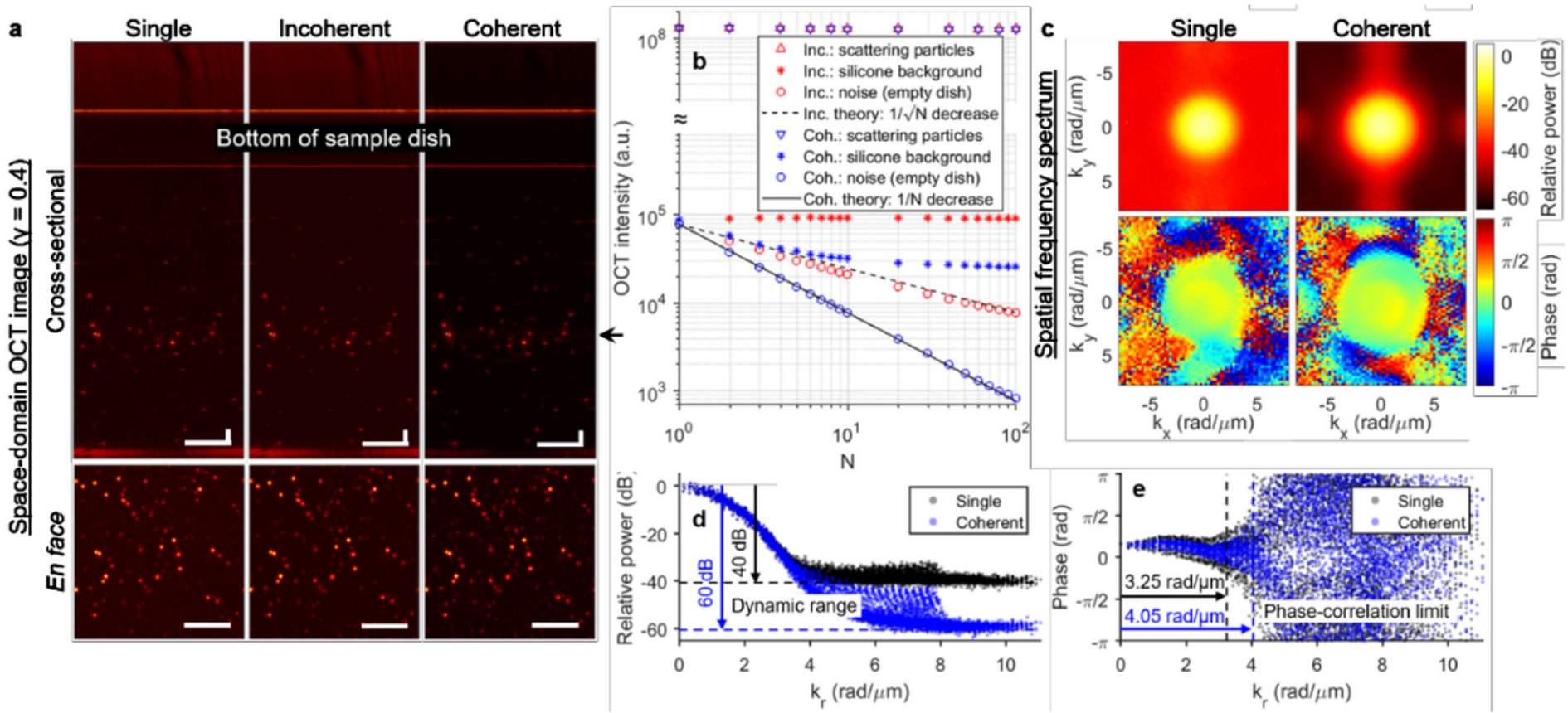

Figure 2

Coherent-average noise suppression with 100 acquisitions in silicone phantom. a, Single-shot, incoherentand coherent-average OCT images (same colormap range). En face images correspond to focal plane, indicated by arrow. Scale bar, $50 \mu \mathrm{m}$. b, OCT intensity of scattering particles, silicone background, and noise as a function of $\mathrm{N}$ for incoherent (red) and coherent (blue) average. Noise intensity was obtained from the "noise image" of an empty sample dish (see Methods), at the same pixel depth as the focal plane of the phantom images. c, Power and phase of single-shot and coherent-average images in transverse spatial-frequency domain (with spatial frequencies kx and ky). Phase spectrum was obtained from the Fourier transform of a windowed region around a single particle. d, Power spectrum in c plotted as a function of radial transverse spatial frequency $\mathrm{kr}$. Dotted line indicates noise floor obtained from power spectrum of the noise image. e, Phase spectrum in c plotted as a function of kr. Dotted line indicates kr limit beyond which phase becomes decorrelated (local standard deviation $>0.2$ rad, see Methods). Supplementary Section VII investigates relations between N, DR and phase-correlation limit. Calculations of OCT intensity, DR, and phase-correlation limit are described in Methods. 
PSF

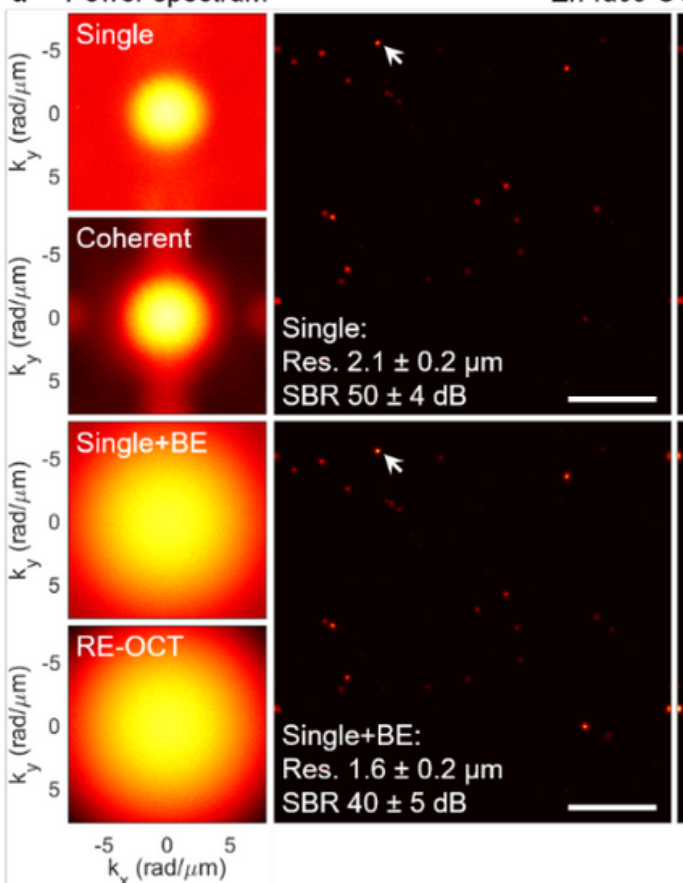

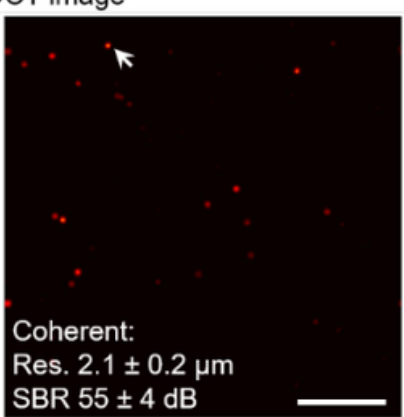

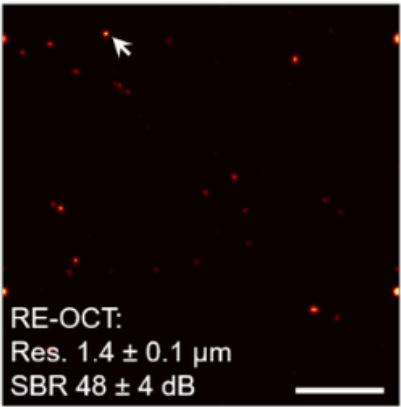

政

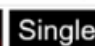

Single
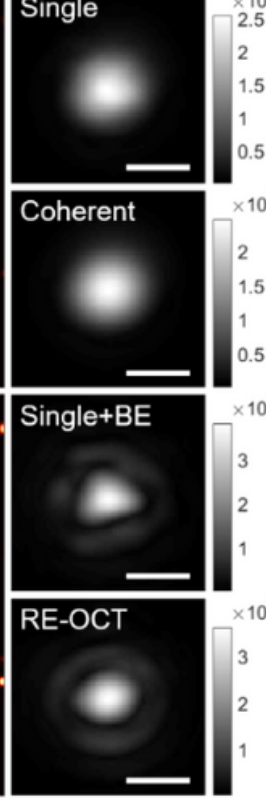
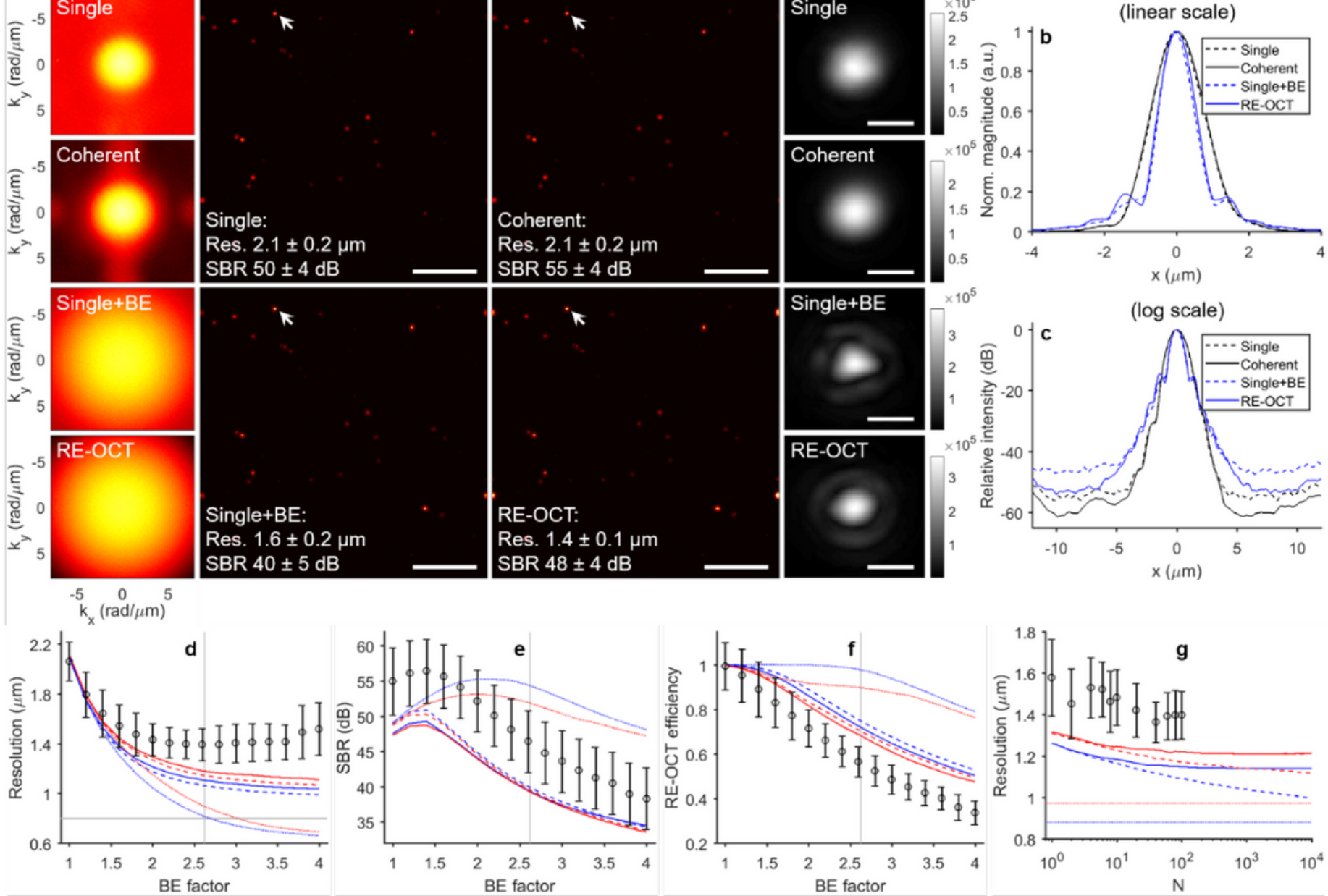

Noise-free limit w/ Ab $\Phi$ Experiment

\section{Figure 3}

RE-OCT with 100 acquisitions and 2.4× bandwidth expansion (BE) in silicone phantom. a, Single-shot, coherent-average, BE single-shot, and RE-OCT power spectrums (log scale) and space-domain en face OCT image with zoomed PSF (linear scale). Resolution and SBR represent mean \pm standard deviation of measurements from 11 particles. Scale bars, $40 \mu \mathrm{m}$ (en face image) and $2 \mu \mathrm{m}$ (zoomed PSF). b and c, Cross-sectional profiles of zoomed PSF in a on peak-normalized linear and log scales. $d-f$, Resolution, SBR, and RE-OCT efficiency as a function of BE factor from experiment and simulations for $\mathrm{N}=100$ of noise-free (dotted), noise only (dashed), and noise with background (solid) conditions, with (red) and without (blue) optical aberrations. Grey vertical lines indicate the Nyquist limit based on spatial sampling of $0.4 \mu \mathrm{m} /$ pixel. g, Resolution as a function of $\mathrm{N}$ from experiment and simulations for BE factor of 2.4 . Resolution and SBR in a and data point and error bars for 'Experiment' in $\mathrm{d}-\mathrm{g}$ represent mean \pm standard deviation of measurements from 11 particles (see Methods). Bk, background. Ab, aberrations. See Supplementary Movie 1 for a movie of $a-c$ as increasing BE factor is applied. 

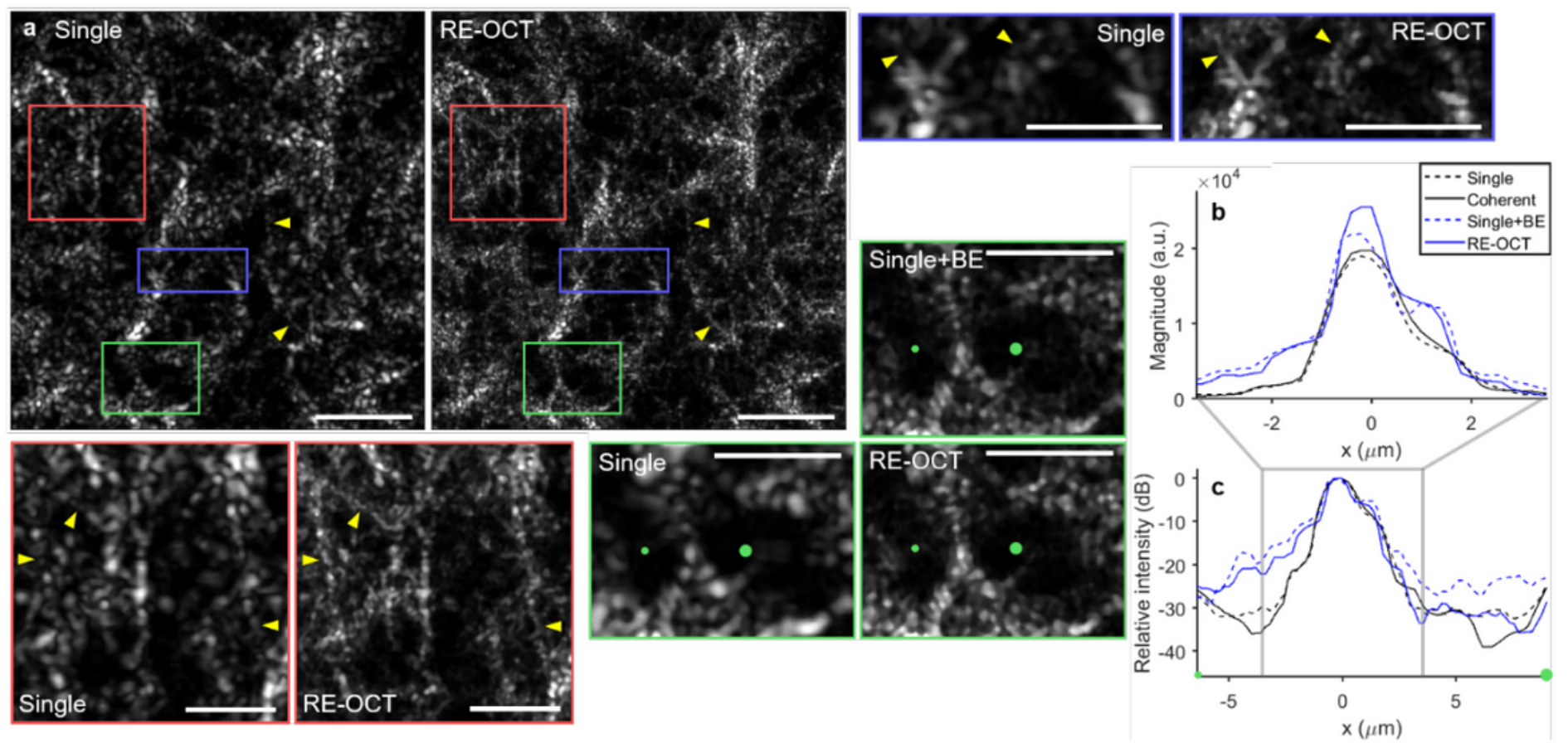

\section{Figure 4}

RE-OCT with 100 acquisitions and 2.0x BE in fibrous collagen gel. a, Single-shot and RE-OCT en face OCT images with zoomed insets regions indicated by boxes. Yellow arrows indicate fine fibre structures that can be more clearly visualized with RE-OCT. Scale bars, $40 \mu \mathrm{m}$ (full) and $20 \mu \mathrm{m}$ (zoomed). b and c, Crosssectional profiles of a line connecting from small to larger green dots in the green zoomed insets in a on linear and peak-normalized log scales. See Supplementary Movie 2 for a movie of a-c as increasing BE factor is applied.
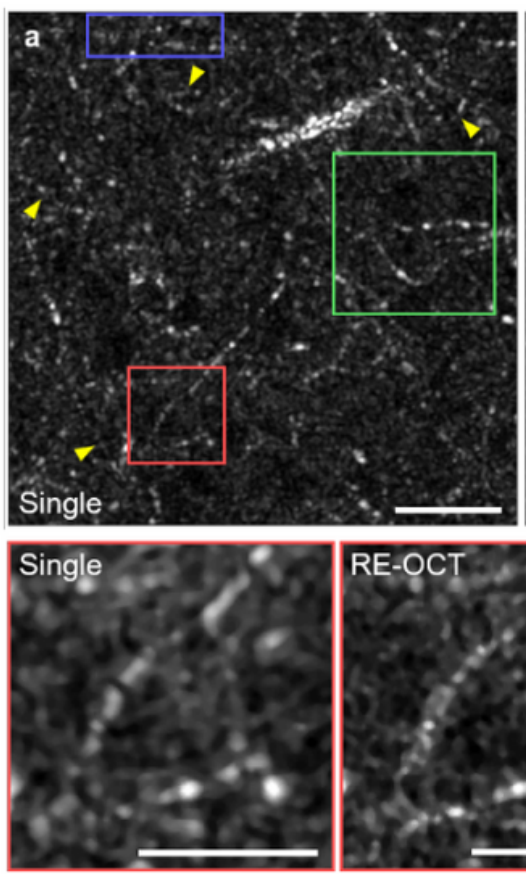
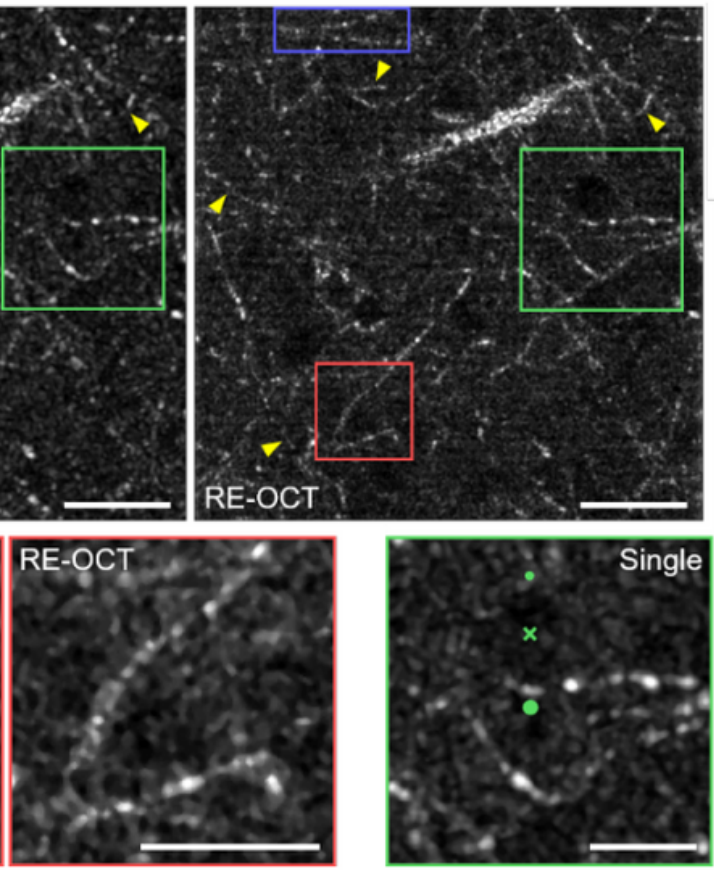
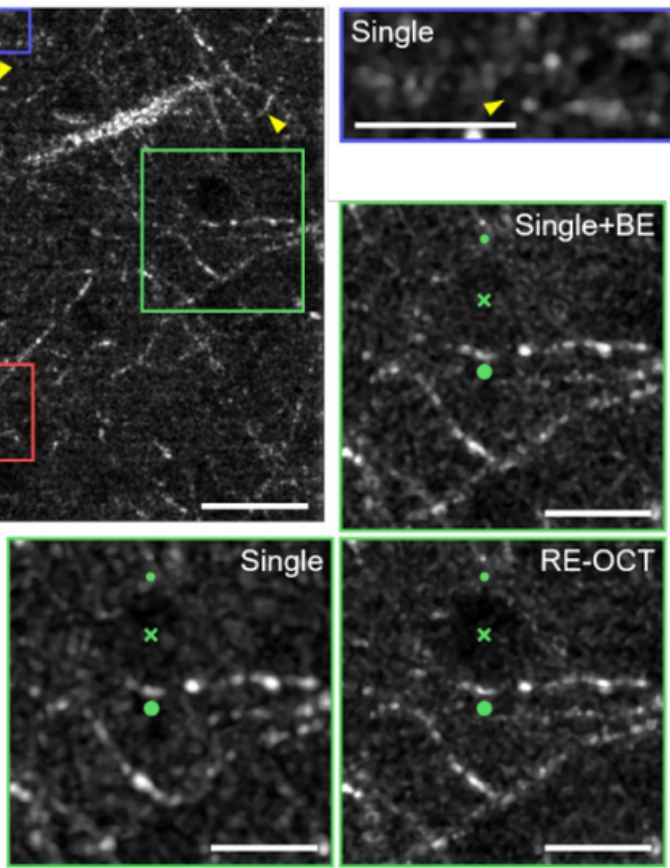
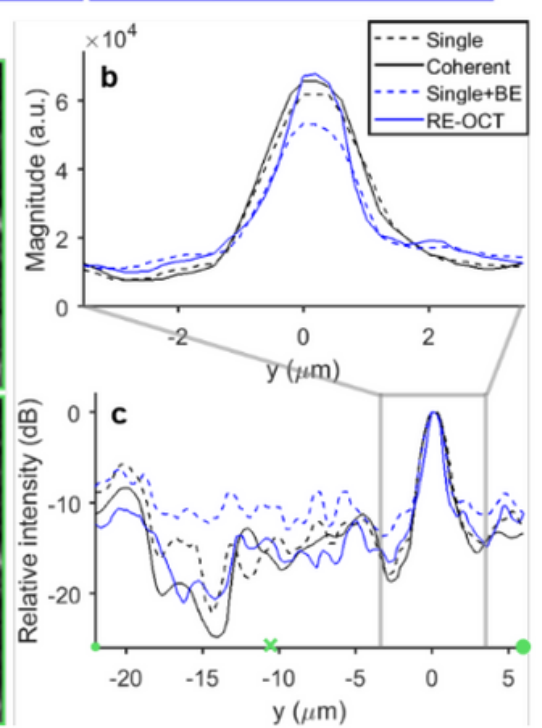


\section{Figure 5}

RE-OCT with 100 acquisitions and 2.0x BE in the cortex of ex vivo mouse brain. a, Single-shot and RE OCT en face OCT images with zoomed insets regions indicated by boxes. Images were taken in the first cortical layer at approximately $100 \mu \mathrm{m}$ below surface. Yellow arrows indicate myelinated axonal processed that can be more clearly visualized with RE-OCT. $\times$ markers in the green zoomed insets indicate one of the neurons, which appear as darker circles due to weak OCT scattering. Green inset shows that neuron in BE single-shot image was barely discernible due to the SNR penalty without coherent-average noise suppression. Scale bars, $40 \mu \mathrm{m}$ (full) and $20 \mu \mathrm{m}$ (zoomed). b and c, Cross-sectional profiles of a line connecting from small to large green dots in the green zoomed insets in a on linear and peaknormalized log scales. The green $\times$ marker indicates its corresponding position on the image. See Supplementary Movie 3 for a movie of a-c as increasing BE factor is applied.

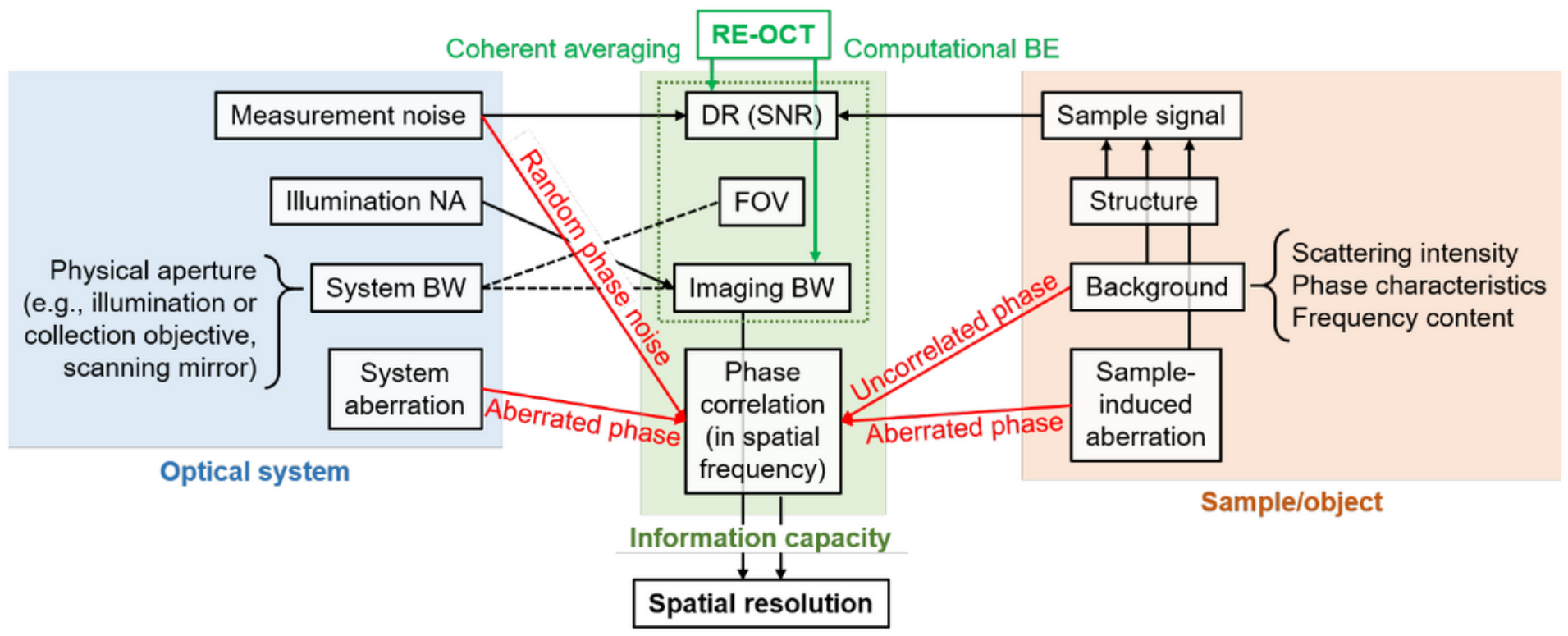

Figure 6

Expanded framework of information capacity and resolution in coherent imaging. Factors that influence elements of the expanded information capacity and ultimately affect resolution in coherent imaging. Phase correlation in the spatial-frequency domain is an important addition to the existing framework of information capacity (green dotted box)4. Red arrows denote factors that can disrupt phase correlation in the spatial-frequency domain. Green arrows indicate the role of RE-OCT: enhancing DR in the spatial frequency domain via coherent-average noise suppression, then, expanding imaging bandwidth via computation BE. For the sake of simplicity, the depicted framework omits the temporal components and only considers information in the spatial dimensions. Supplementary Section VII discusses the relationship between system and imaging bandwidths, illumination NA, and FOV. BW, bandwidth.

\section{Supplementary Files}


This is a list of supplementary files associated with this preprint. Click to download.

- LeartprapunREOCTSciRepMovieS1.mp4

- LeartprapunREOCTSciRepMovieS2.mp4

- LeartprapunREOCTSciRepMovieS3.mp4

- LeartprapunREOCTSciRepSupplementaryInformation.docx 